Article

\title{
Design Analysis of a Passive Buffer System for Space-Assembled Segmented Mirrors
}

\author{
Xupeng Li ${ }^{1,2}$, Wei Wang ${ }^{1}$, Jinfeng Shi ${ }^{1, *}$, Yanjun Qu ${ }^{1,2}$ and Xuewu Fan ${ }^{1}$ \\ 1 Xi'an Institute of Optics and Precision Mechanics, Chinese Academy of Sciences, Xi'an 710119, China; \\ lixupeng@opt.cn (X.L.); wangwei@opt.ac.cn (W.W.); quyanjun@opt.cn (Y.Q.); fanxuewu@opt.ac.cn (X.F.) \\ 2 University of Chinese Academy of Sciences, Beijing 100049, China \\ * Correspondence: shijinfeng@opt.ac.cn
}

Received: 25 February 2019; Accepted: 6 March 2019; Published: 8 March 2019

\begin{abstract}
This paper presents a passive buffer system of connection mechanism for space-assembled segmented mirrors. The purpose of assembling telescope in space is to overcome the limits of launch volume and mass to support ever increasing aperture sizes. Using this buffer system, the docking impact between two mirror segments can be alleviated effectively. In this paper, first, a design methodology called "double-elastic contact model" is developed. Then, the design details and operation principle of the connection mechanism, are presented to validate the passive buffer system. Finally, by comparing the traditional docking system, the simulated results of dynamic performance show that the proposed buffer system can absorb the impact energy effectively and is considerably suitable for assembling the space optical system.
\end{abstract}

Keywords: passive buffer system; space assembled telescope; double-elastic contact model; dynamic performance

\section{Introduction}

With the continuous advancement of human exploration of the universe, the demand for higher optical resolution has driven the aerospace observatory moving forward to the larger aperture ultraviolet-optical-infrared (UVOIR) space telescopes, with the primary mirror (PM) segmented [1,2]. The prospective space assembled telescope [3-6] will enable optical elements to be launched in batches, compared with traditional space optical telescopes employing monolithic optics [7]. Therefore, future space observation systems will overcome the launching mass and volume limitations. However, a critical issue is effectively capturing and connecting the precise optical mirror segments in space to determine the optical stability of the telescope structure [8].

The connection technology applied between primary mirror assemblies (PMAs) means that two or more modular units are connected rigidly by locking mechanisms, with special docking interfaces to form the core of the synthetic aperture. Then, the co-focal and co-phase adjustment operations are executed with actuators attached to the backside of the individual mirror segments, thereby directly contributing to the optical performance. The space probe-cone docking mechanism is ideally suitable for the space small-scale cooperative target self-assembly tasks, mainly due to the high reliability of capture, high docking precision, and very compact structure. Because of the microgravity environment in space, a strong coupling effect exists between free-floating space objects in the docking process. Therefore, a buffer system is essential to alleviating the influence of the docking impact and controlling the contact force. However, considering the size compactness and power efficiency, it is difficult for complex active buffer systems to join this type of docking mechanism. In other words, if there is a simple solution to mitigate the impact coupling effectively during the assembly period, the probability for assembling PMAs on orbit will be greatly improved. 
At present, many organizations [9-12] are conducting research on connection technology using space small-scale targets. The autonomous micro-satellite docking system (AMDS) $[13,14]$, developed by the Orbital Express (OE) project, utilized soft-docking technology [15] to buffer the impact. However, the docking conditions of this technology were harsh, undermining the overall capture rate. The Engineering Test Satellite VII (ETS-VII) [16] demonstrated autonomous rendezvous and docking experiments in space. The universal docking port (UDP), developed by the Synchronized Position Hold Engage Reorient Experimental Satellites (SPHERES) team [17], adopted the electromagnetic techniques $[18,19]$ for active buffering. Its operation was difficult and energy consumption could not be ignored.

In this paper, a novel design methodology named "double-elastic contact model," is mainly developed for the passive buffer system between the modular PMAs to assemble one large-scale apertures. A connection mechanism called the Self-configurable Modular Adjustable Latch Lock (SMALL) is provided, using the passive buffer system to mitigate the impact. Through the universal explicit probe-cone interface, this simple and compact design contributes immensely to segmented mirrors to function monolithically. This paper is organized as follows. Section 2 analyzes the general capture process of the autonomous probe-cone docking mechanism for modular assembled mirror segments. And the "double-elastic contact model" is investigated in detail. In Section 3, a passive buffer system applied to the connection mechanism SMALL is implemented and analyzed to verify the theory mentioned in Section 2. Section 5 concludes our statements.

\section{Establishment of Double-Elastic Contact Model}

According to the mission requirements of the space assembled telescope, and the collision characteristics of the probe-cone connection mechanism, the docking process between PMAs should be divided as follows.

1. First collision. Within a reasonable capture range, two PMAs could achieve a capture operation of weak impact, preventing strong vibrations and interference of the mirrors.

2. Positioning. It is necessary to limit partial degrees of freedom (DOFs) between two PMAs to align and steady the combination.

3. Locking and Adjustment. A mechanically rigid connection between the optical elements is required. At the same time, the ability of mirror gap adjustment is a pre-requisite in improving optical performance.

4. Separation. The feasible undocking maneuver of the connection mechanism should be satisfied in case of unpredictable incidents, such as abnormal docking or irreversible collision.

The buffer performance design mainly focuses on the first point mentioned above, because it produces the largest impact influence, which means the buffer mechanism should be required to absorb the impact energy.

In our previous studies of the typical small-sized cooperative target collision system [20], the rigid protruding pin $\mathrm{B}$ made the first contact with the rigid chamfered entrance hole $\mathrm{A}$, during the capture stage as shown in Figure 1. The conditions for successful capture by analysis are:

$$
\left\{\begin{array}{c}
\varepsilon_{1}<90^{\circ}-2 \beta \\
V_{n}^{1}=S V_{n}^{0} \\
V_{\tau}^{1}=V_{\tau}^{0}+(1+S) T V_{n}^{0} \\
T=M \sum_{i}^{2}\left(\frac{\sin \mu}{m_{i}}+\frac{r_{i}^{2} \cos \theta_{i} \sin \left(\theta_{i}-\mu\right)}{I_{i z}}\right) \quad(i=A, B)
\end{array}\right.
$$

where $\beta$ represents the half cone angle of the chamfered hole $A$; the entrance angle, and the exit angle of the pin $B$, are represented by $\varepsilon_{0}, \varepsilon_{1}$, respectively. The normal and tangential velocities of the contact point before impact are shown as $V_{n}^{0}, V_{\tau}^{0}$, respectively. Similarly, $V_{n}^{1}, V_{\tau}^{1}$ represent the normal and 
tangential velocities of the contact point after impact. In addition, the velocity ratio before, and after, the collision is defined as the recovery factor $S$; $T$ stands for the equivalent stiffness; $M$ is expressed by the equivalent mass of the connection mechanism; the coefficient of friction angle of conical surface is shown as $\mu$; the angle between the vector of the two docking mechanisms centroid to collision point and the normal of the cone is expressed by $\theta ; I_{i z}$ is the moment of inertia around the $Z$ axis of the mechanism.

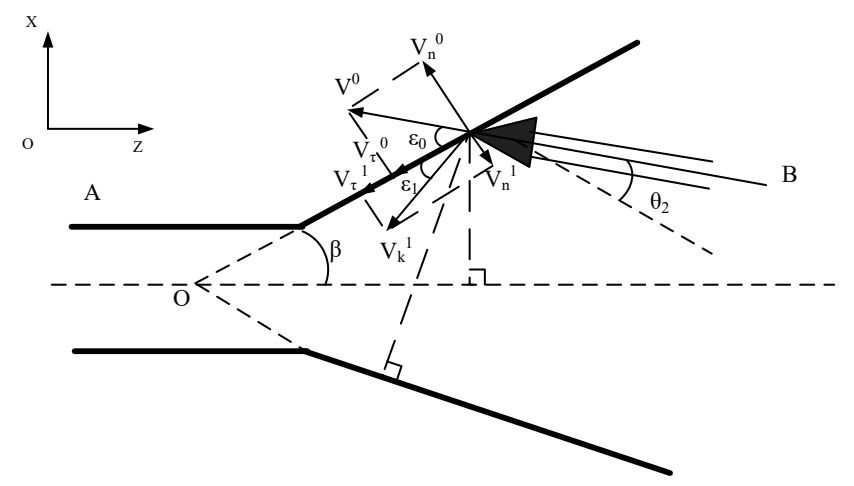

Figure 1. The traditional study of the contact between the pin and the chamfered hole, both of which are considered rigid.

According to Equation (1), in the case of successful capture, the relationship between the velocity of the rigid pin and the rigid inner-wall of the hole can be obtained by:

$$
\tan \varepsilon_{1}=\frac{S}{\frac{1}{\tan \varepsilon_{0}}+(1+S) T}<\tan \left(90^{\circ}-2 \beta\right) .
$$

However, in the actual space on-orbit assembly environment, a rigid collision can cause irreversible damage to the mirror, such as vibration and interference damage. Therefore, a buffer system needs to be added to mitigate the effects of impact during the docking process. To minimize the energy requirements on such a large cluster of PMAs processing, a passive buffer system, rather than an electromagnet driving one, was developed in this paper. For this purpose, "double-elastic contact model" is established to satisfy this buffer system.

To satisfy the optical specifications and ensure the impact force absorption, storage, and consumption, a passive buffer system was designed and analyzed. On the basis of the structural shape, the pin and the inner-wall of the entrance hole are re-designed flexibly in the probe-cone docking system. In this study, the "double-elastic contact model" was established to study the collision, between the elastic head of the protruding pin and the elastic inner-wall of the entrance hole, during the capture stage. This model is helpful in analyzing whether the maximum deformation and maximum compressive stress meet the assembly requirements of the on-orbit telescope.

Under the double-elastic contact model, Figure 2 illustrates the details of transient docking collision where the elastic head of the protruding pin $B$ collides with the elastic inner-wall of conical hole $A$ with non-zero incident angle $\varepsilon_{0}$ and velocity $v_{0}$ in the position $i$. The maximum deformation $\delta$ is produced on the contact surface of $A$ when the pin head of $B$ moves to the position $i i$. The pin head leaves the elastic wall at the position $i i i$ through the exit angle $\varepsilon_{1}$ and exit velocity $v_{k}$. At position $i i$, centered on the collision point $O$, the sectional view can be obtained in the plane perpendicular to the common normal of the collision point, as shown in the red region of Figure 2. Since the deformation $\delta$, caused by the contact, is much smaller than the radius of the elastic inner-wall $R_{a}$ and the pin head $R_{b}$, it is assumed that the radius $R_{1}$ is expressed by $R_{1}=R_{a} / \cos \beta$ and the spherical cone head radius is $R_{2}=R_{b}$. Therefore, the contact pressure and contact deformation will be discussed. 


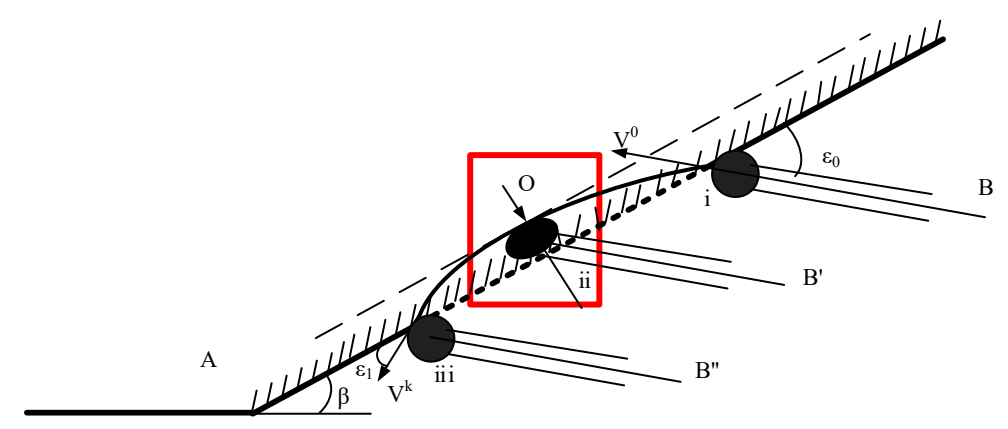

Figure 2. Transient collision of docking connectors with an elastic pin head engaging with an elastic inner-wall of a chamfered hole.

As Figure 3 shows, during capture stage, it is assumed that the elastic pin head collides with the elastic inner-wall, at point $O$ under the driving force $F$. A local coordinate system $O X Y$ is established with the contact point $O$ as the origin. The $X$-axis, and $Y$-axis, represent the common normal, and tangential direction of the collision location, respectively. It is assumed that two points $C\left(x_{1}, \xi\right)$ and $D\left(x_{2}, \xi\right)$ are taken arbitrarily and will coincide to anyone point within the contact surface during the collision. Then according to the geometric relationship, it can be obtained that

$$
\left\{\begin{array}{l}
x_{1}=\frac{\xi^{2}}{2 R_{1}} \\
x_{2}=\frac{\tilde{\xi}^{2}}{2 R_{2}}
\end{array} .\right.
$$

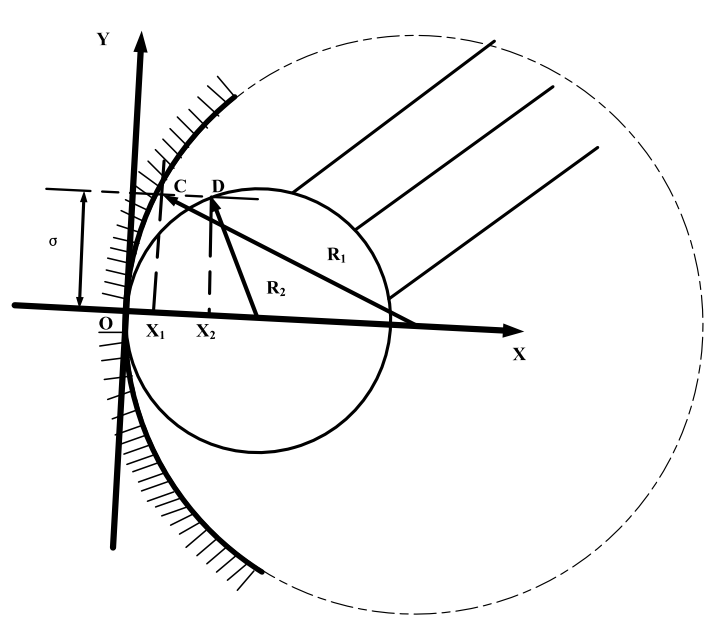

Figure 3. The detailed contact model. And this figure shows the sectional view of the red region in Figure 2.

Therefore, the deformation $\delta$ of the collision can be represented as:

$$
\delta=\left(x_{2}-x_{1}\right)+\left(e_{2}-e_{1}\right),
$$

where $e_{1}$ and $e_{2}$ represent the displacement of deformation generated by the point $C$ and the point $D$ during the collision, respectively.

From the Equations (3) and (4), the deformation $\delta$ can be written by

$$
\left\{\begin{array}{l}
\delta=\gamma \xi^{2}+k \iint f d s d \psi \\
\gamma=\frac{R_{1}-R_{2}}{2 R_{1} R_{2}} \\
k=\frac{1-v_{2}^{2}}{\pi E_{2}}+\frac{1-v_{1}^{2}}{\pi E_{1}}
\end{array},\right.
$$


where $E_{1}, E_{2}$, and $v_{1}, v_{2}$ represent the Young's modulus and Poisson's ratio of the elastic inner-wall of $A$, and the elastic head of $B$, respectively. $f$ is shown as a compressive stress function, whose integral area is the entire contact surface.

According to the Hertz theory of elastic mechanics, the compressive stress function, $f$ can be solved by the semi-inverse solution method. Assuming that the compressive stress function $f$ on the contact circle of radius $u$ is proportional to the ordinate of the hemispherical surface, so we can get:

$$
k \frac{\pi^{2} f_{0}}{4 u}\left(2 u^{2}-\xi^{2}\right)=\delta-\gamma \xi^{2}
$$

By comparing the coefficients on both sides of the Equation (6), it can be easily seen that Equation (6) can be rearranged as:

$$
\left\{\begin{array}{l}
\delta=k \frac{\pi^{2} u f_{0}}{2} \\
\gamma=k \frac{\pi^{2} f_{0}}{4 u}
\end{array},\right.
$$

where $f_{0}$ is represented as the maximum compressive stress on the contact circle.

According to the equilibrium condition, the product of the volume of the hemisphere mentioned above and $\frac{f_{0}}{u}$ should be equal to the driving force, which can be shown as:

$$
\frac{f_{0}}{u} \frac{2}{3} \pi u^{3}=F
$$

From Equations (7) and (8), the radius $u$ of contact circle and the maximum deformation $\delta$ can be obtained by:

$$
\left\{\begin{array}{l}
u=\left[\frac{3 \pi F k R_{1} R_{2}}{4\left(R_{1}+R_{2}\right)}\right]^{\frac{1}{3}} \\
\delta=\left[\frac{9 \pi^{2} F^{2} k^{2}\left(R_{1}+R_{2}\right)}{16 R_{1} R_{2}}\right]^{\frac{1}{3}} .
\end{array}\right.
$$

The maximum compressive stress $f_{0}$ occurs at the center of the contact surface, and its value is:

$$
f_{0}=\frac{3 F}{2 \pi}\left[\frac{4\left(R_{1}+R_{2}\right)}{3 \pi F k R_{1} R_{2}}\right]^{\frac{2}{3}}
$$

Since $k$ is related to the Young's modulus $E$, and the Poisson's ratio $v$ of the elastic pin head and the elastic wall surface, the value of the maximum compressive stress $f_{0}$ can be determined as long as we know the wall radius, $R_{1}$ at the collision point $O$, and the magnitude of the driving force.

\section{Buffer System Validation}

At present, one of the important factors restricting the development of on-orbit PM assembly is how to connect the modular PMAs, which can ensure that the assembled telescope achieve optical performance requirements. The mechanical strength and precision need to be satisfied between PMAs, so the design of the connection mechanism plays an important role. In this section, the proposed double-elastic contact model is considered for the androgynous connection mechanism, SMALL. This modulization scheme is a critical conceptual upgrade attached to the optical elements, as illustrated in Figure 4.

It is predominantly comprised of: A chamfered entrance hole and a grooved protruding pin which increase docking tolerances; a positioning channel to correct alignment error by limiting five degrees of freedom (DOFs); a latch lock to connect two adjacent modules rigidly; and a linear actuator to control the clearance between combined parts. This compact and simple interface is attached to the outer wall of the PMA base, which solves the defects, such as high energy consumption, high operation difficulty, insufficient locking force, and low adjustment precision, compared with the traditional connection mechanism. 


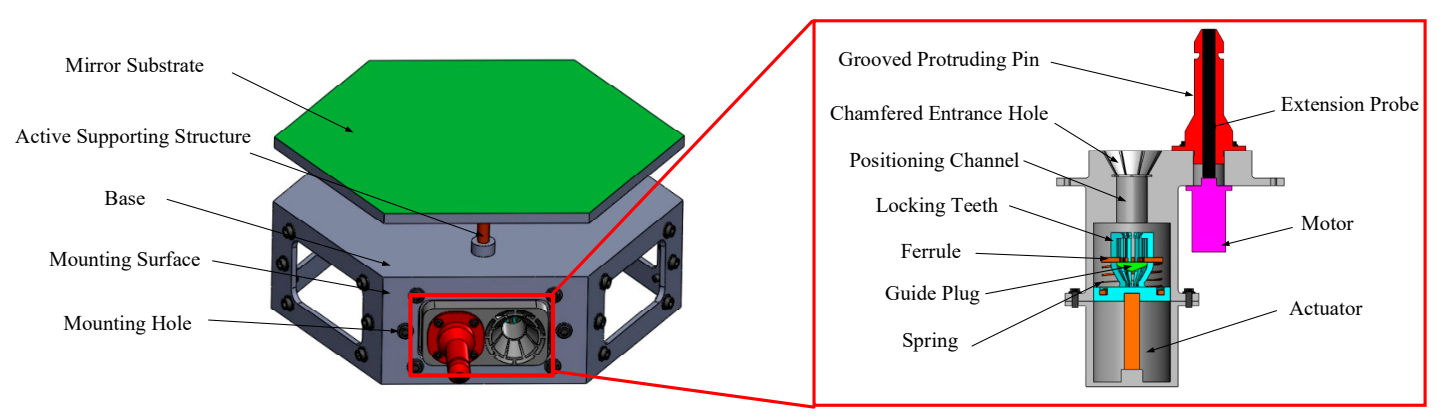

Figure 4. Introduction of a modular PMA (left) and the design scheme of SMALL (right).

The operation principle of SMALL is explained as shown in Figure 5. In the capture stage, under the monitoring of the camera, two SMALLs are simultaneously close to each other. The pins will make contact with the opposing holes, as shown in Figure 5a. During the positioning stage, the pins enter the positioning channels to limit five DOFs of the combination. Only one DOF is left along the axis hole direction, as shown in Figure 5b. In the locking and adjustment stage, the groove of the pin engages with the locking mechanism, which has twelve locking teeth [21]. After the lock is completed, the internal actuators of SMALLs start to align the gap between the two mirror segments to a reasonable range, as shown in Figure 5c. In the separation stage, the locking teeth expand outwards, with the assistance of the extended semi-rigid stick within the grooved pin, separating the two connection mechanisms from each other as shown in Figure 5d.

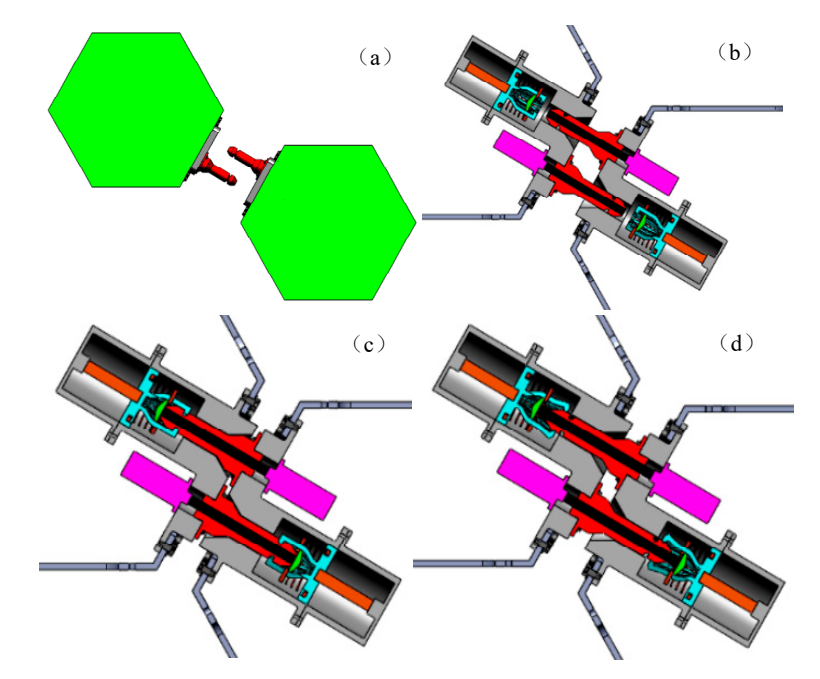

Figure 5. Operation principle of SMALL: (a) capture, (b) positioning, (c) locking \& adjustment, (d) separation.

The SMALL adopts a flexible design of the inner-wall of the tapered cone. Eight flexure sections are evenly distributed on the chamfered surface. As shown in Figure 4, the gap between the flexible sections and the tapered surface is consistent. According to the design requirements, the deformation of the buffer system should be within $0.002 \mathrm{~m}$ according to the material properties and structural characteristics.

Taking the actual working environment into account, it is assumed that the driving force is with the range of $100 \mathrm{~N} 500 \mathrm{~N}$, and the protruding pin and the inner-wall of the hole are made of TC4. The docking parameter specifications are shown in Table 1. 
Table 1. The docking parameter specifications.

\begin{tabular}{cc}
\hline Parameter & Value \\
\hline Young modulus E $(\mathrm{Pa})$ & $1.09 \times 10^{11}$ \\
Poisson's ratio $\mathrm{V}$ & 0.34 \\
The radius of the pin head $(\mathrm{m})$ & 0.020 \\
The depth of the conical hole $(\mathrm{m})$ & 0.020 \\
Half-cone angle $\left(^{\circ}\right)$ & 60 \\
\hline
\end{tabular}

According to the double-elastic model, the deformation and compressive stress can be acquired from

$$
\left\{\begin{array}{l}
\delta=1.99 e-7\left[\frac{F^{2}\left(0.02+R_{1}\right)}{R_{1}}\right]^{\frac{1}{3}} \\
f_{0}=0.12 e 9\left[\frac{F\left(0.02+R_{1}\right)^{2}}{R_{1}^{2}}\right]^{\frac{1}{3}} .
\end{array}\right.
$$

Depending on the location of the collision point, the value of the $R_{1}$ is changing. Assuming that the $Z$-axis is along with the center axis of the conical hole, and the propulsion depth of the pin head is denoted by $z$, the elastic pin head makes the first collision with the elastic inner wall. Then there is:

$$
R_{1}=\frac{(0.020-z) \tan \beta}{\cos \beta}
$$

With the different values of $F$, the maximum deformation and the maximum compressive stress are shown below.

The line graph, illustrated in Figure 6, demonstrates the relationship between the maximum collision deformation and the driving force under different depths of propulsion. It is obvious that the deformation value rises considerably as the driving force increases from $100 \mathrm{~N}$ to $500 \mathrm{~N}$, revealing that the buffer system has a good absorption of impact energy. As shown in the curve, the deformation at $\mathrm{Z}=0.020 \mathrm{~m}$ is far beyond the others, generally appearing in the span from $1 \times 10^{5} \mathrm{~m}$ to $3 \times 10^{5} \mathrm{~m}$, which shows the effect of deformation on the bottom of the chamfered hole is evident. However, it is necessary to point out that the deformation value still does not exceed the specified requirements.

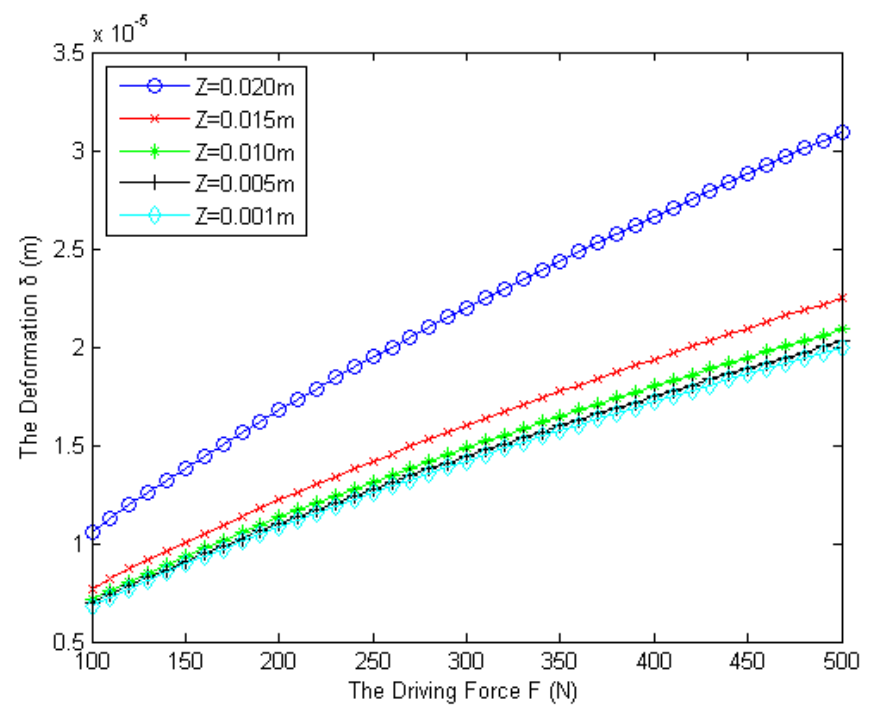

Figure 6. The relationship between the driving force and the maximum deformation under different depths of propulsion. In this, to make the graph easier to read, trend lines have been added. 
The relationship between the maximum compressive stress and the driving force is delivered by Figure 7. The rising trends of the compressive stress, under different depths of propulsion, always keep at a slow rate, showing this system is very effective in damping the impact force. Although the compressive stress remains in a secure range, it is worth mentioning that the line at $Z=0.020 \mathrm{~m}$ is still much higher than the others, going up from $1.5 \times 10^{9} \mathrm{~Pa}$ to $2.5 \times 10^{9} \mathrm{~Pa}$. Therefore, efforts should be made to avoid the contact position too close to the bottom of the chamfered hole, in case of damage to the PMAs.

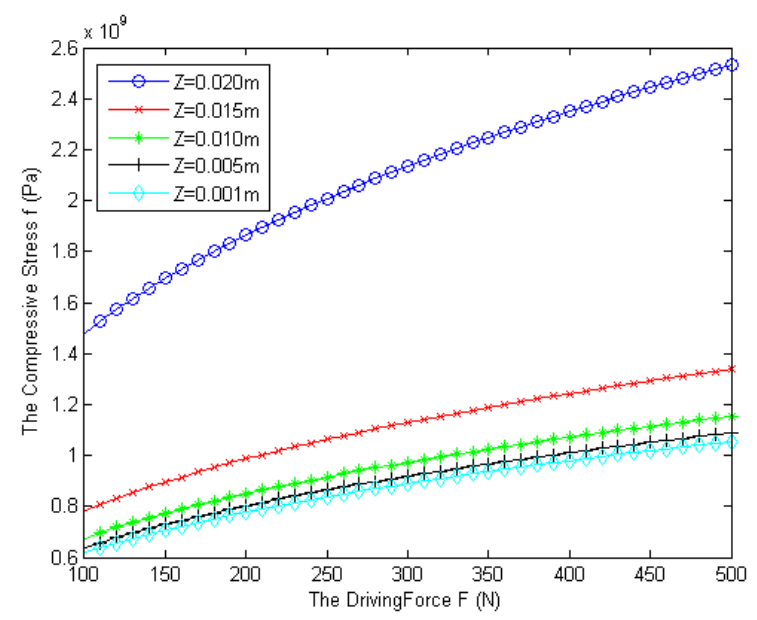

Figure 7. The relationship between the driving force and the maximum compressive stress under different depths of propulsion.

\section{Simulation Analysis}

In order to test and verify the buffer ability of the proposed theory, there should be evaluation to assess whether the capture contact meets the requirements of optical assembly. Due to a lack of experiment environment and equipment, it is an effective method for the dynamic simulation on computer to validate the parameters of the buffer system designed.

A stack of parameters with zero gravity environment were chosen to compare the magnitude of the collision, such as axial docking velocity, lateral deviation, and roll angular velocity. One PMA is designed to remain stationary and stable at the center of the simulated environment. Meanwhile, another PMA approaches and connects with it via the parameterized docking maneuver. Referring to docking data from Automated Rendezvous and Docking of Spacecraft [22] and SPHERES docking experiment [23], typical initial docking conditions of SMALL are listed in Table 2.

Table 2. Initial docking conditions of SMALL.

\begin{tabular}{cccccc}
\hline Parameters & $\begin{array}{c}\text { Axial Docking } \\
\text { Velocity }(\mathbf{m m} / \mathbf{s})\end{array}$ & $\begin{array}{c}\text { Maximum Lateral } \\
\text { Bias Error }(\mathbf{m m})\end{array}$ & $\begin{array}{c}\text { Roll Angular } \\
\text { Velocity }(\% / \mathbf{s})\end{array}$ & $\begin{array}{c}\text { Pitch/Yaw Angular } \\
\text { Velocity }\left({ }^{\circ} / \mathbf{s}\right)\end{array}$ & $\begin{array}{c}\text { Roll Bias Error } \\
\left({ }^{\circ}\right)\end{array}$ \\
\hline Value & 5 & 10 & $-1 \sim 1$ & $-1 \sim 1$ & $-10 \sim 10$ \\
\hline
\end{tabular}

The thrust value is set to $100 \mathrm{~N}$. Taking the androgyny characteristic of SMALL into account, there will be two contact positions for the probe-cone docking process. Due to the existence of the docking deviation, there is always a case where a set of pin and hole is contacted, and then another set is contacted. Under the above conditions, the docking simulation between the two PMAs is performed by Adams software. The contact force diagram of a typical rigid collision system is shown as Figure 8 . Likewise, that of the double-elastic collision system is shown as Figure 9. 


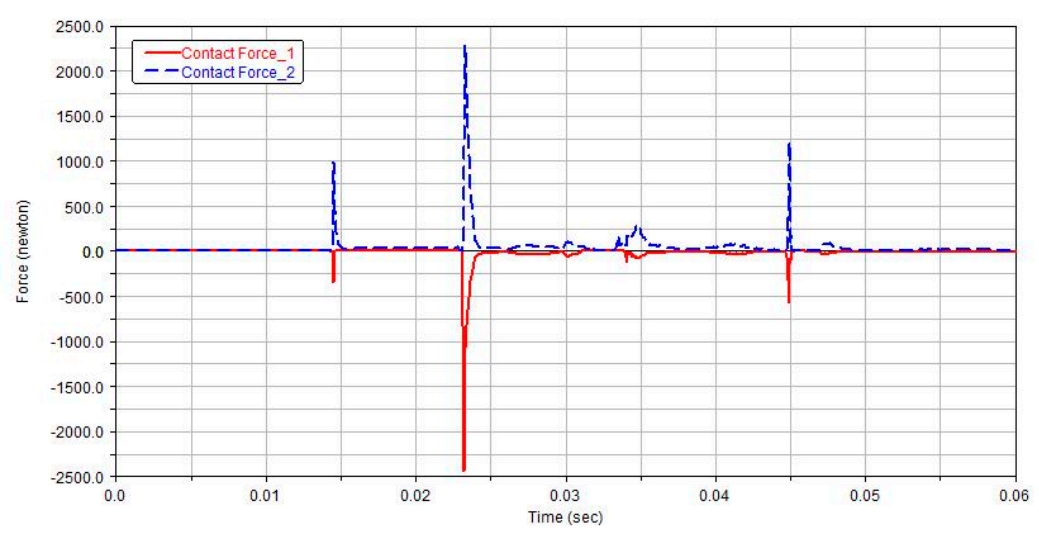

Figure 8. The contact force of a typical rigid collision system. The red line indicates the contact force between the first set of pin and hole while the blue line indicates the contact force between the second group.

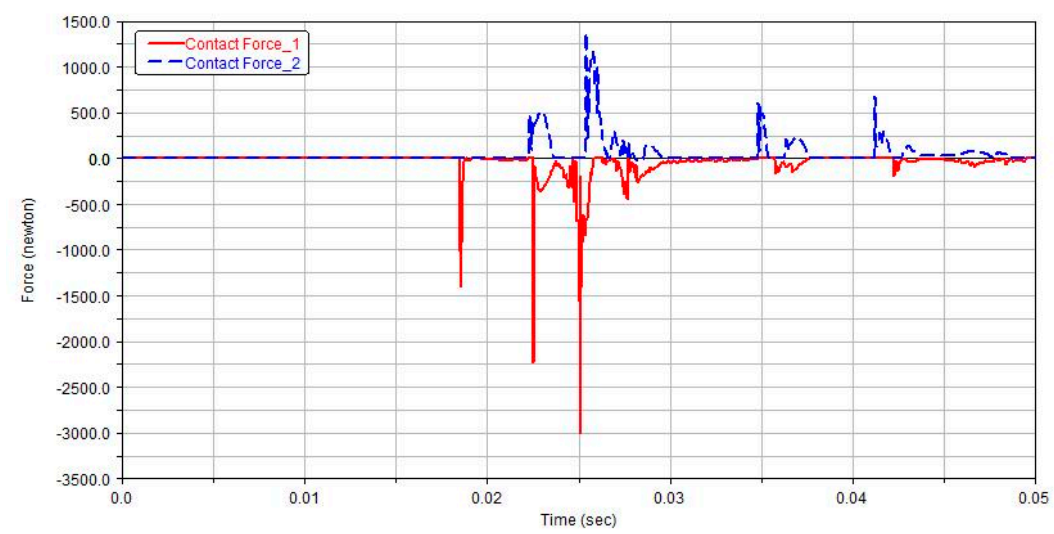

Figure 9. The contact force of the double-elastic collision system. The red line indicates the contact force between the first set of pin and hole while the blue line indicates the contact force between the second group.

Under the same docking conditions, the contact forces between the first set are almost the same, as the red lines shows in Figures 8 and 9. However, by comparison, it should be noted that the contact force of the second set of pins and holes produced a significant change. The connection mechanism, using the passive buffer system, generates a collision force much smaller than that of the conventional un-buffered system. The contact force decay rate under the double-elastic contact model is $58 \%$, which is much larger than $10 \%$ under the traditional rigid collision. Therefore, it can be concluded that the double-elastic contact model is feasible.

\section{Conclusions}

In this paper, a passive buffer system for the space-assembled optical telescope is investigated. Through a theoretical research, the "double-elastic contact model" of the probe-cone docking mechanism in the capture stage, is established and the solution of the maximum contact compressive stress, and the maximum buffer deformation, are also derived. Based on this model, a design, and implementation of a passive buffer system is presented in which, both the pin and the cone wall, are elastically designed. Compared with the small-sized traditional docking mechanism, the dynamic simulation results prove that the impact energy is well-absorbed by increasing the deformation of the flexible structure. The proposed connection mechanism SMALL, using this buffer system, is light-weight, compact, and considerably power efficient, which can meet the needs of on-orbit assembled telescope very well. 
Author Contributions: Conceptualization, X.L. and W.W.; Methodology, X.L.; Software, Y.Q.; Validation, X.L., W.W. and Y.Q.; Formal Analysis, X.L.; Investigation, X.L.; Resources, X.L. and F.S.; Data Curation, X.L.; Writing-Original Draft Preparation, X.L.; Writing-Review \& Editing, X.L.; Visualization, W.W.; Supervision, W.W. and X.F.; Project Administration, F.S.; Funding Acquisition, F.S.

Funding: This research and the APC was funded by West Light Foundation of The Chinese Academy of Sciences grant number [XAB2016B24].

Acknowledgments: The authors would like to thank three PHD candidates, Bei Liu, Chenchen Wang and Yang Shen in University of Chinese Academy of Sciences, for their enthusiastic participation in our research.

Conflicts of Interest: The authors declare no conflict of interest.

\section{References}

1. Izzo, D.; Pettazzi, L.; Ayre, M. Mission concept for autonomous on orbit assembly of a large reflector in space. In Proceedings of the 56th International Astronautical Congress, Fukuoka, Japan, 17-21 October 2005.

2. Lightsey, P.A.; Atkinson, C.B.; Clampin, M.C.; Feinberg, L.D. James Webb Space Telescope: Large deployable cryogenic telescope in space. Opt. Eng. 2012, 51, 011003. [CrossRef]

3. Gralla, E.L.; De Weck, O. On-Orbit Assembly Strategies for Human Space Exploration. In Proceedings of the 56th International Astronautical Congress, Fukuoka, Japan, 17 October-21 October 2005.

4. Postman, M.; Brown, T.M.; Sembach, K.R.; Tumlinson, J.; Mountain, C.M.; Soummer, R.; Oegerle, W.R. Advanced technology large-aperture space telescope: Science drivers and technology developments. Opt. Eng. 2012, 51, 011007. [CrossRef]

5. Feinberg, L.D.; Budinoff, J.G.; MacEwen, H.A.; Matthews, G.W.; Postman, M. Modular assembled space telescope. Opt. Eng. 2013, 52, 091802. [CrossRef]

6. Carpenter, K.G.; Etemad, S.; McElwain, M.; Seery, B.D.; Thronson, H.; Burdick, G.M.; Liu, F. OpTIIX: An ISS-Based Testbed Paving the Roadmap toward a Next Generation, Large Aperture UV/optical Space Telescope. Available online: file:/ / C:/Users/MDPI/AppData/Local/Temp/12-2435-2.pdf (accessed on 10 February 2019).

7. Williams, R.E. The Hubble Deep Field: Observations, data reduction, and galaxy photometry. arXiv 1996, arXiv:astro-ph/9607174.

8. Lillie, C.F. Large deployable telescopes for future space observatories. In UV/Optical/IR Space Telescopes: Innovative Technologies and Concepts II, Proceedings of the SPIE-The International Society for Optical Engineering, San Diego, CA, USA, 31 July 2005; International Society for Optics and Photonics: Bellingham, WA, USA.

9. Nella, J. James Webb Space Telescope (JWST) observatory architecture and performance. In Proceedings of the Space 2004 Conference and Exhibit, San Diego, CA, USA, 28-30 September 2004.

10. Basu, S.; Mast, T.; Miyata, G. A proposed autonomously assembled space telescope (AAST). In Proceedings of the AIAA Space 2003 Conference Exposition, Long Beach, CA, USA, 23-25 September 2003.

11. Akin, D.; Roberts, R.; Lane, J.; Weisman, S. Robotic capabilities for complex space operations. In Proceedings of the AIAA Space 2001 Conference and Exposition, Albuquerque, NM, USA, 28-30 August 2001.

12. LeMaster, E.; Schaechter, D.; Carrington, C. Experimental Demonstration of Technologies for Autonomous On-Orbit Robotic Assembly. In Proceedings of the Space 2006, San Jose, CA, USA, 9-21 September 2006.

13. Han, W.; Huang, Y.; Chen, X. Research of impact dynamic modeling of flexible probe-cone docking mechanism based on Kane method. Arch. Appl. Mech. 2015, 85, 205-221. [CrossRef]

14. Hays, A.B.; Tchoryk, P.; Pavlich, J.C.; Ritter, G.A.; Wassick, G.J. Advancements in design of an autonomous satellite docking system. In Proceedings of the Spacecraft Platforms and Infrastructure International Society for Optics and Photonics, Orlando, FL, USA, 30 August 2004.

15. Hays, A.; Tchoryk, P.; Pavlich, J. Dynamic simulation and validation of a satellite docking system, in Space Systems Technology and Operations. Int. Soc. Opt. Photonics 2003, 5088, 77-89.

16. Kawano, I.; Mokuno, M.; Kasai, T.; Suzuki, T. Result and evaluation of autonomous rendezvous docking experiment of ETS-VII. In Proceedings of the Guidance, Navigation, and Control Conference and Exhibit, Portland, OR, USA, 9-11 August 1999.

17. Otero, A.S.; Chen, A.; Miller, D.W.; Hilstad, M. SPHERES: Development of an ISS laboratory for formation flight and docking research. In Proceedings of the IEEE Aerospace Conference, Big Sky, MT, USA, 9-16 March 2002; Volume 1. 
18. Mohan, S. Reconfiguration Methods for On-Orbit Servicing, Assembly, and Operations with Application to Space Telescopes. Doctoral Dissertation, Massachusetts Institute of Technology, Cambridge, MA, USA, 2007.

19. Rodgers, L.; Hoff, N.; Jordan, E.; Heiman, M.; Miller, D. A Universal Interface for Modular Spacecraft. Available online: https://digitalcommons.usu.edu/smallsat/2005/all2005/4/ (accessed on 15 February 2019).

20. Li, X.; Wang, W.; Shi, J. Design, analysis of self-configurable modular adjustable latch lock for segmented space mirrors. Opt. Express 2018, 26, 18064-18081. [CrossRef] [PubMed]

21. Li, X.; Wang, W.; Shi, J. Design and analysis of a novel connective locking mechanism for future space mirrors assembly, in Micro-Optics and MOEMS. Int. Soc. Opt. Photonics 2018, 10848, 108480D.

22. Fehse, W. Automated Rendezvous and Docking of Spacecraft; Cambridge University, Cambridge University Press: New York, NY, USA, 2003.

23. InSPIRE-II Program ISS Science Report, InSPIRE-II ISS Science Report. $2016 . \quad$ Available online: http:/ /ssl.mit.edu./spheres/spheresLibrary/documents / projectDocumentation/InSPIRE-II_ISS_ Science_Report.pdf (accessed on 22 September 2017).

(C) 2019 by the authors. Licensee MDPI, Basel, Switzerland. This article is an open access article distributed under the terms and conditions of the Creative Commons Attribution (CC BY) license (http:/ / creativecommons.org/licenses/by/4.0/). 